Sociologie et sociétés

\title{
Institutions financières, allocation du crédit et marginalisation dans le Nord-Est du Brésil \\ L'exemple de Bahia
}

\section{Financial Institutions, Credit Allocation and Marginalization in the Brazilian Northeast The Bahian Case}

\section{Harry M. MAKLER}

Volume 11, numéro 2, octobre 1979

Développement national et économie mondialisée

URI : https://id.erudit.org/iderudit/001471ar

DOI : https://doi.org/10.7202/001471ar

Aller au sommaire du numéro

\section{Éditeur(s)}

Les Presses de l'Université de Montréal

ISSN

0038-030X (imprimé)

1492-1375 (numérique)

Découvrir la revue

Citer cet article

MAKLER, H. M. (1979). Institutions financières, allocation du crédit et marginalisation dans le Nord-Est du Brésil : l'exemple de Bahia. Sociologie et sociétés, 11(2), 145-168. https://doi.org/10.7202/001471ar

\section{Résumé de l'article}

Cet article constitue une étude empirique des pratiques discriminatoires imposées par les banques à la bourgeoisie industrielle et à leurs entreprises dans l'État du Nord-Est du Brésil qui se développe aujourd'hui le plus vite. Fondé sur une enquête par interviews auprès d'un échantillon aléatoire et systématique des dirigeants des plus grandes entreprises, ainsi que sur des données statistiques provenant des archives gouvernementales et bancaires cet essai établit l'histoire économique et politique de cet État et les problèmes qui ont entravé son émergence. Une analyse des opérations de crédit, des emprunts à court et à long terme, et de taux d'intérêts montre que les banques d'État, en particulier la Banque du Brésil, marginalisent la bourgeoisie industrielle associée aux secteurs économiques traditionnels et aux entreprises familiales. Elles appuient, par contre, les dirigeants des firmes nouvelles, à haute intensité de capital qui sont souvent des filiales de grands conglomérats nationaux ou internationaux. L'une des conséquences inattendues de l'intervention de l'État et de l'hégémonie du capital financier est la pénétration du capital étranger dans les secteurs à haute intensité de capital. Ceci peut éroder le soutien et le sacrifice populaires sur lesquels le régime compte pour poursuivre son miracle économique. Cet essai montre ainsi comment les institutions clés sont utilisées par l'État pour stratifier et contrôler les classes sociales dans une des zones au développement le plus rapide en Amérique latine et peut être dans le Tiers Monde.
Tous droits réservés @ Les Presses de l'Université de Montréal, 1979
Ce document est protégé par la loi sur le droit d'auteur. L’utilisation des services d'Érudit (y compris la reproduction) est assujettie à sa politique d'utilisation que vous pouvez consulter en ligne.

https://apropos.erudit.org/fr/usagers/politique-dutilisation/ 


\section{Institutions financières, allocation du crédit et marginalisation dans le Nord-Est du Brésil : l'exemple de Bahia*}

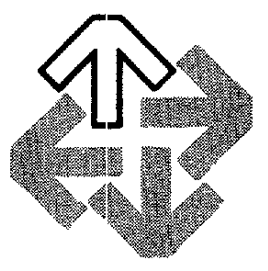

HARRY MARK MAKLER

\section{INTRODUCTION}

Au Brésil, les efforts en vue de maintenir le taux de croissance et le modèle de développement ont conduit à des innovations financières et à de nouvelles alliances de groupes sociaux qui ont une influence marquée sur l'économie: et la société. Les marchés financiers nationaux et internationaux, ainsi que les changements institutionnels ont été conçus principalement pour mobiliser plus efficacement les ressources intérieures et étrangères pour atteindre des

* Version remaniée de la communication au colloque sur «les implications sociales et politiques du nouvel ordre économique international » organisé par le Comité de recherche «Economie et société" de l'Association internationale de sociologie, fondation Rockefeller à Bellagio, Italie, 24-28 avril 1979. C'est grâce à l'aide généreuse de l'International Development Research Centre, Canada Council, du comité conjoint Social Science Research Council - American Council of Learned Societies et du Humanities and Social Sciences Comittee, la Banque du Nord-Est, l'Université de Toronto que cette recherche a pu être réalisée. Je remercie aussi Andrew Beveridge, Richard Bird, Rondo Cameron, Barry Ames, Antonio Barros de Castro, Emilio F. Moran, Arnaud Sales, Neil Smelser et Charles Wagley pour leurs commentaires et en particulier Wendy Barker pour les modifications qu'il a suggérées. La version anglaise originale paraîtra dans Makler, Martinelli et Smelser, édit., The New Transnational Economic Order, Sage Publications. Pour des raisons d'espace, quelques paragraphes, plusieurs notes et tous les tableaux de cet article ont été soustraits, malgré leur intérêt. Le lecteur peut s'adresser à l'auteur pour les obtenir. (Note de l'éditeur.) 
objectifs de développement déterminés. Les études sur la question n'ont pas suffisamment souligné le rôle des institutions financières dans la répartition réelle des ressources entre certaines classes, certaines régions et certains secteurs de l'économie. Parmi toutes les nations en voie de développement, le Brésil fournit un cadre optimal pour examiner l'impact des institutions financières sur la structure des classes et sur la croissance économique. C'est surtout depuis 1964 que le Brésil a réagi au contexte international et qu'il a commencé à promouvoir sa croissance en instituant de nouveaux mécanismes mettant à contribution ses institutions financières, dans une stratégie destinée à rattraper les nations les plus développées du monde. Nous espérons pouvoir soulever certaines questions, sinon y répondre, au sujet de l'effet de ces développements sur la structure de classe dans le Nord-Est brésilien, région de l'Amérique latine qui a subi les transformations les plus rapides, en particulier Bahia qui est la cible d'une intervention et d'une industrialisation massives de la part de l'État ${ }^{1}$.

L' «intermediation» financière - c'est-à-dire la mobilisation et la distribution du surplus de capital des épargnants aux investisseurs dans les divers secteurs de l'économie en fonction du taux de rendement - constitue une activité socioéconomique à la fois importante et révélatrice. Qui prend les décisions au cours de ces opérations, comment et à qui sont alloués les crédits et à quelles fins? Les réponses à ces questions sont extrêmement utiles pour expliquer la stratification sociale et le développement économique. Nous allons montrer comment le mode de distribution du crédit peut accélérer le développement de certains secteurs de l'économie de Bahia que l'État considère comme vitaux pour atteindre ses objectifs de développement. Mais en même temps ces interventions ralentissent la croissance économique de certains secteurs «moins dynamiques» et des classes sociales qui leur sont liées, en restreignant leur accès aux capitaux disponibles de manière à freiner certaines activités économiques.

Depuis 1964, le régime brésilien a décrété et poursuivi délibérément une politique économique «developpementiste». Les stratégies financières mises en œuvre par l'État montrent clairement que son objectif est de réorienter l'économie vers l'industrialisation et de réorganiser les structures sociales pour y parvenir. En fait, l'État a joué le rôle d'intermédiaire financier. La réalisation de ce discours politique lui assure une grande part de sa crédibilité et de sa légitimité. Le jeu pourrait se définir par: «l'efficacité avant l'égalité», soit, la modernisation aux dépens des inégalités structurales (chômage élevé, grande concentration des revenus, et comme nous le verrons, marginalisation des entreprises familiales traditionnelles.) Compte tenu de cette idéologie «développementiste», la prospérité ne profite qu'à une bourgeoisie économique représentant un faible pourcentage de la population, alors que le niveau de vie de la majorité n'augmente à peu près pas, car la mobilité sociale se heurte à de

1. David E. Goodman et Roberto Cavalcanti de Albuquerque, Incentivos a Industrializaçâo e Desenvolvimento do Nordeste, Rio de Janeiro, Instituto de Planejamento Econômico e Social, 1974, p. 283-287. Voir également Albert O. Hirschman, «Industrial Development in the Brasilian Northeast and the Tax Credit Scheme of Article 34/18\%, dans A Bias for Hope, New Haven, Yale University Press, 1971, p. 142-145. 
sévères restrictions, tandis que la participation politique des masses est jugulée. La base productive du développement économique est générée de l'extérieur et monopolisée par la bourgeoisie internationale; ainsi le régime répressif n'est pas mis en cause, car il fait croire que le taux élevé de croissance économique profite à tout le monde sans exception.

Il se peut que par leur nature et par leur origine, les investissements qui alimentent ce projet entrent en conflit avec les intérêts et les résistances de divers groupes marginalisés de la société, mais en fin de compte, c'est celui qui manipule le "robinet" des investissements qui va orienter et dominer l'économie, et perpétuer les contradictions inhérentes au système. Or les flux de capitaux les plus importants sont controlés d'une part de l'extérieur par un réseau bancaire international, et d'autre part, à l'intérieur, par un État qui sert les intérêts de classe d'une bourgeoisie industrielle intimement liée au capitalisme international; d'où le renforcement de la dépendance historique du Brésil vis-àvis de l'étranger.

Malgré l'intention déclarée de l'État de renforcer le secteur privé, l'accroissement de son intervention depuis 1964 dans l'industrie et l'infrastructure s'est faite aux dépens du secteur privé. Le rôle de l'entreprise privée continue d'être subordonné à celui de l'État dans l'allocation des ressources financières qui favorisent le schéma actuel de croissance économique. Comme l'État a monopolisé les secteurs les plus modernes et les plus rentables de l'économie, il a limité le champ d'action des entreprises privées, ce qui accentue la faiblesse historique de ce secteur. Il ne peut faire concurrence aux entreprises relativement efficaces de l'État, ni avoir accès comme lui aux capitaux des réseaux bancaires internationaux qui préfèrent consentir des prêts à longue échéance à des gouvernements «solvables». Les entreprises publiques assurant des placements relativement sûrs et d'un haut rendement attirent plus facilement les actionnaires que le secteur privé. Ces caractéristiques se reflètent dans les taux d'intérêt, et nous allons montrer comment les banques utilisent ce mécanisme pour la répartition efficace des ressources financières aux industries dynamiques dont le rendement est le plus profitable. Il semble peu probable que le secteur privé puisse atteindre un niveau économique, technologique et financier nécessaire au succès dans la grande industrie sans avoir accès à un financement bon marché et à longue échéance. Sur le plan régional, nous allons montrer comment certaines industries et certains entrepreneurs du secteur privé ne peuvent obtenir que des prêts à court terme qui suffisent comme capital de roulement (ex. pour les salaires), mais non pour l'expansion et la croissance. En fait, on peut montrer comment des banques peuvent «étouffer» certaines industries.

\section{HISTORIQUE}

Dans le Nord-Est brésilien, l'industrie s'est développée en fonction de structures résultant de la formation d'intermédiaires financiers spécifiques, et non l'inverse. Même vers la fin des années 50, le Nord-Est était caractérisé par une économie d'exportation de matières premières stagnante. Les industries, 
à propriété familiale, étaient de petite taille et liées aux secteurs d'exportation primaire, ou à la production de biens non durables pour le marché local.

La croissance industrielle n'exigeait pas un grand afflux de capitaux, et le secteur financier ne jouait pas un rôle primordial dans l'exportation des matières premières. Les exportateurs utilisaient leurs propres capitaux, ou bien bénéficiaient de lettres de crédit des importateurs. Certaines des plus importantes entreprises d'exportation étaient sous contrôle étranger (ex., SANBRA) ou des sociétés à participation étrangère (ex., Cimento Aratu) ${ }^{2}$. Aussi, le secteur financier avait-il tendance à restreindre son activité et ses investissements au développement du commerce (garanties d'import/export) et des infrastructures. Pour répondre aux besoins financiers, il suffisait d'un réseau bancaire relativement simple qui n'alimentait ni l'agriculture, ni l'industrie. Cette situation engendra des pratiques qui allaient sérieusement modifier l'économie du Nord-Est au cours des années suivantes. Premièrement, les surplus de capitaux du NordEst furent transférés vers les pôles de croissance du Centre-Sud du pays qui promettaient des bénéfices plus élevés. Deuxièment, à ces transferts volontaires sont venus s'ajouter des transferts involontaires résultant des politiques d'échange du gouvernement national qui transféraient les surplus de capitaux de l'exportation agricole du Nord-Est vers les secteurs industriels naissants du CentreSud. Il en est résulté une augmentation chronique du déficit commercial, surtout avec le Centre-Sud, et une fuite de capitaux de l'ordre de plus de $\$ 413$ millions de dollars durant les années 50 , soit, en moyenne, plus de $\$ 38$ millions par an. Afin d'assurer l'accumulation du capital, le gouvernement utilisait des mesures «parafiscales» pour remplacer un système bancaire incapable de financer la transition d'une économie d'exportation de matières premières à une économie urbaine industrialisée. Ce problème s'est particulièrement aggravé au cours de la période de substitution des importations (1962-1964) quand des investissements à grande échelle et à longue échéance se sont avérés nécessaires ${ }^{3}$.

Ce qui est important, de notre point de vue, ce ne sont pas seulement les sorties de capitaux - il faudra bien les remplacer par des rentrées, un jour, pour redonner du dynamisme à l'économie du Nord-Est - mais c'est la logique qui sous-tend ce mouvement. Les mesures fiscales permettaient au gouvernement de se substituer aux institutions financières du Centre-Sud qui ne pouvaient pas, ou ne voulaient pas fournir le capital nécessaire à une économie en transition. Le même problème va se présenter plus tard dans le Nord-Est, mais dans ce cas, il ne sera pas question de se passer des institutions financières. Au contraire le gouvernement les utilisera comme leviers pour tirer le Nord-Est de son état de stagnation.

Pour plusieurs, le développement économique se conçoit comme une lente croissance des épargnes et des investissements selon une trajectoire unilinéaire.

2. Clemente Mariani, «Análise do Problema Económico Baiano», O Observador Económico e Financeiro, 267, mai 1958, p. 19.

3. Le Mexique est l'un des rares pays de l'Amérique latine où le système des institutions financières était suffisamment développé pour répondre aux exigences d'une croissance rapide. Voir Maria Conceiçâo Tavares, "Financianento Numa Economia em Desenvolvimento», $D a$ Substituiçâo de Importaçôes ao Capitalismo Financeiro, Rio de Janeiro, Zahar Editores, 1973, p. 129. 
En fait, il nécessite l'adoption de modèles économiques pouvant exiger un réaménagement des institutions financières, afin de mieux mobiliser les surplus de capitaux, surtout pour transférer des ressources à de nouveaux secteurs. Cependant, le secteur financier du Nord-Est avait pour principale fonction de soutenir l'économie d'exportation des matières premières. Certaines banques étaient même la propriété de l'élite agro-commerciale qui considérait sa position menacée par la croissance de l'industrie de substitution des importations ${ }^{4}$.

Donc, dans la concurrence pour le partage des maigres ressources financières, le secteur industriel naissant fut défavorisé au profit de projets plus conventionnels tels que l'expansion de l'infrastructure qui permettait aux principaux centres urbains d'intégrer un hinterland de plus en plus vaste dans sa sphère d'influence commerciale. La bourgeoisie agro-commerciale et la bourgeoisie financière résistèrent à ce nouvel ordre industriel, et les principales victimes de cet état de choses furent les directeurs des nouvelles entreprises de biens durables destinés aux consommateurs.

Il fallait en fait à ces nouveaux entrepreneurs un capital financier considérable qui manquait dans le Nord-Est. Ce besoin était exacerbé par deux autres facteurs : leur dépendance vis-à-vis de la technologie étrangère et la nécessité de satisfaire la demande croissante d'une classe moyenne en expansion ${ }^{5}$. Tout ceci se produisait dans le Nord-Est au moment où l'existence de structures financières archaïques ne permettait pas aux institutions de mobiliser les ressources nécessaires, et où le capital excédentaire était drainé vers les régions plus prospères du pays. Plus les industriels dépendaient du secteur financier, moins le secteur financier pouvait répondre à leus exigences ${ }^{6}$.

Il semble que les problèmes liés à cette première période de développement du Nord-Est se soient répercutés dans la structure financière contemporaine, selon deux modèles apparemment opposés. En premier lieu, et d'après mes données, la bourgeoisie industrielle est à la remorque des banques. À Bahia, $65 \%$ de ceux qui furent interrogés déclarèrent qu'ils finançaient leurs activités surtout grâce à des crédits bancaires. En éliminant ceux qui utilisent des crédits non bancaires et ceux qui n'ont pas répondu à notre question, seulement $29 \%$ des industriels de Bahia ont surtout recours à l'autofinancement, et parmi ceux-là, la participation d'associés privés représentait un pourcentage encore moindre ${ }^{7}$. Peu de compagnies émettent des actions pour obtenir du capital. C'est une pratique qui exige l'existence d'une solide classe moyenne ayant des revenus suffisamment élevés, ainsi que d'un assez grand nombre d'entreprises disposant de la confiance du public et désireuses d'émettre des actions. Bien

4. Liens et interdépendances entre les secteurs bancaires et agricoles caractérisant le NordEst durant les années 50. Voir, par exemple, Antônio Barros de Castro: Sete Ensaios Sobre a Economia Brasileira, São Paulo, Compania Editôra Forense, 1971, vol. II, p. 216-219.

5. Ibid. p. 242-244.

6. Maria Conceição Tavares, op. cit. p. 128-130 et surtout 138-139, «Financiamento Numa Economia em Desenvolvimento», Da Substituiçâo de Importaçôes ao Capitalismo Financeiro, Rio de Janeiro, Zahar Editores, 1973, p. 129.

7. Luciano Martins dans son article sur les «joint-ventures» de l'État et des transnationales à Bahia, présenté dans ce même numéro, trouve aussi que comparativement à l'État la participation financière des partenaires privés est «extrêmement faible». 
qu'une Bourse ait été instituée récemment au Brésil (avec un succès mitigé) il y eut peu d'investissements sous forme de capital action dans les entreprises du Nord-Est. Beaucoup d'industriels l'ont reconnu, ainsi que les réponses suivantes l'indiquent :

La Bourse ici, contrairement aux États-Unis, n'a pas eu de succès. Le marché des valeurs mobilières n'existe qu'à São Paulo où les investisseurs n'achètent que des actions des grandes entreprises du Sud comme Volkswagen. Ou bien: Ça ne sert à rien d'émettre des actions, vu que le public ne les achète pas.

Cependant, quand il était question de leurs projets de financement, un bon nombre (soit $34 \%$ ) avouaient que s'ils développaient leurs entreprises, ils préféreraient avoir recours au capital action. Ce n'est pas la volonté qui manque, ce sont les conditions favorables.

$39 \%$ seulement de la bourgeoisie industrielle utilisaient fréquemment les banques du secteur privé, et une plus faible proportion encore leur empruntait du capital. Cela s'explique par une pénurie de capital dans les banques, et par leur impossibilité à consentir des crédits à moyen ou à long terme et beaucoup d'industriels s'en sont plaint.

Si l'on soustrait du secteur bancaire privé les banques dont le siège social est à l'extérieur de la région, le pourcentage des prêts consentis s'en trouve diminué d'autant. Le crédit fourni par les banques privées du Nord-Est est de l'ordre de $19 \%$. En tenant compte de l'acquisition récente de la plus grande banque privée du Nord-Est, la Banco de Bahia par une banque du Sud (Bradesco), ce pourcentage est réduit de plus de la moitié.

Bref, le secteur bancaire privé n'a pas su répondre aux exigences du secteur industriel, et cela, particulièrement dans le Nord-Est. Si l'on songe que Salvador, la capitale de Bahia, fut un des principaux centres bancaires du Brésil, cette décadence est d'autant plus remarquable.

Cela s'explique par deux tendances. D'abord, les banques privées du NordEst se font éclipser par les banques privées du Sud ou de l'étranger, soit par l'établissement de succursales, soit par des fusions. Ensuite, tout le système bancaire privé est progressivement éliminé par les banques d'État ${ }^{8}$, en même temps que croissent les besoins du secteur industriel en ressources financières.

$\mathrm{Au}$ moyen de ses institutions financières (et même des banques du Sud) le régime tente de se substituer aux anciennes banques du Nord-Est qui n'ont pas su répondre aux besoins d'un secteur industriel dynamique. Ceci implique la création de banques de développement aux niveaux du pays, de la région et de l'État, et l'intervention dans les activités bancaires commerciales ainsi que dans les programmes d'industrialisation. Les meilleurs exemples de ce phénomène sont les programmes de la Superintendance pour le Développement du NordEst (SUDENE) et de la Banque du Nord-Est (BNB). La création du SUDENE en 1959, pour administrer un programme régional de développement «global intégré» a surtout stimulé le développement industriel, grâce à l'Article 34/18,

8. Ce sujet est amplement étudié par Maria Conceição Tavares, op. cit., p. 223-230. 
une puissante mesure de dégrèvement fiscal ${ }^{9}$. L'autre facteur de développement, c'est la Banque du Nord-Est (BNB) qui finance des programmes à un taux d'intérêt très favorable (soit $12 \%$ par an sans indexation à l'inflation, comparativement à 30 ou 40 pour cent dans les banques commerciales) et ce crédit peut constituer jusqu'à 50 pour cent du capital supplémentaire nécessaire à l'entrepreneur pour réaliser son projet ${ }^{10}$. Si l'on tient compte du taux d'inflation au Brésil, ces prêts ne représentent aucun frais, et même un bénéfice. Outre ces alvantages fiscaux et financiers offerts par le gouvernement, chaque État du Nord-Est, et même chaque grande ville de ces États ont mis en place des programmes pour attirer de nouvelles industries ' ${ }^{1}$. À Pernambuco, par exemple, il y a la Banco de Desenvolvimento do Pernambuco (BANDEPE) et à Bahia, une institution semblable s'appelle la Banco de Desenvolvimento do Estado de Bahia (DESENBANCO) ${ }^{12}$.

À l'exception peut-être de l'Amazone, le Nord-Est a été la région du Brésil la plus favorisée du point de vue des programmes d'industrialisation et du financement. Cependant cette intervention ne peut pas être considérée comme une simple réponse à des besoins industriels; l'implication du gouvernement atteirit un degré tel que la relation entre la demande et la réponse est en fait inversée. Ainsi l'État, par son intervention, est-il devenu le promoteur d'un ordre industriel en émergence. Grâce à son rôle prépondérant dans le financement de l'industrie, il détermine non seulement son niveau (celui du développement industriel atteint jusqu'ici) mais également sa structure (soit l'équilibre entre les secteurs industriels et leur distribution).

\section{LA BOURGEOISIE INDUSTRIELLE DE BAHIA ET SES RAP- PORTS AVEC LES INSTITUTIONS FINANCIÈRES}

\section{A. ANALYSE}

Une enquête faite en 1973 et 1974 et portant sur un échantillon $(\mathrm{N}=134)$ de chefs d'entreprise de Bahia ${ }^{13}$ fournit les données nécessaires à l'étude des

9. L'article $34 / 18$ permet aux compagnies brésiliennes de déduire 50 pour cent de leur impôt sur le revenu annuel, si elles s'engagent à investir dans des projets approuvés par SUDENE dans le Nord-Est. SUDENE peut mettre en cuvre de nombreux autres stimulants à l'investissement, comme des exemptions d'impôt pour dix ans ou la suppression des droits de douane sur les machines et autres marchandises importées. Voir notamment, Albert O. Hierschman, Journeys Toward Progress, Garden City, New York, Anchor-Doubleday, 1965, p. 126-129 et aussi «Industrial Development in the Brazilian Northeast and the Tax Credit Scheme of article 34/18 ", Journal of Development Studies, 5, octobre 1968, pp. 5-28.

10. David E. Goodman et Roberto de Albuquerque, Incentivos a Industrializaçâo e Desenvolvimento do Nordeste, Rio de Janeiro, Instituto de Planejamento Económico e Social, 1974, p. 195-217.

11. Ibid., p. 199

12. La plupart des fonds de ces banques proviennent des impôts des grandes entreprises d'État, des dépôts privés à terme fixe et d'emprunts sur le marché national ou étranger (voir, par exemple, Wilson Suzigan et al, Financiamento de Projectos Industriais no Brasil, Rio de Janeiro, Instituto de Planejamento Económico e Social, 1972, p. 166 et 174-180.

13. Les données proviennent d'interviews menées auprès des présidents de toutes les entreprises de 200 salariés et plus et d'un échantillon d'entreprises ayant entre 50 et 199 salariés. 
questions concernant le contrôle de l'État sur l'industrialisation du Nord-Est et la structure de classe induite à travers l'«intermediation» financière.

La principale variable est l'origine géographique de la bourgeoisie industrielle : il peut s'agir d'autochtones du Nord-Est; de migrants venus d'autres régions du Brésil (principalement du Centre-Sud) ou d'étrangers (nés à l'étranger). Ces catégories correspondent à peu près à l'émergence du système de stratification au sein de la classe industrielle aux différents stades de développement de Nord-Est en général, et de Bahia en particulier. Elles représentent également un ensemble de valeurs bien définies des caractéristiques d'entreprise, telles que le secteur industriel, la propriété familiale ${ }^{14}$, la complexité de la technologie ${ }^{15}$, la taille de l'entreprise ${ }^{16}$, ses liens de parenté avec d'autres firmes, la participation du capital étranger ${ }^{17}$, et sur le plan biographique, l'origine sociale des industriels ${ }^{18}$. Ainsi, les autochtones représentent un stade antérieur du développement économique lié aux secteurs traditionnels (aliments, boissons, tabac, textiles, produits du bois et du papier, imprimerie et lithographie) soit des entreprises familiales à haute intensité de main d'œuvre. Beaucoup de ces industriels étaient d'origine socio-économique aisée et avaient hérité de leurs entreprises. Plus tard, ce groupe fut évincé (quoiqu'en termes relatifs) par des migrants de la classe moyenne, du type gestionnaire, et issus des économies plus modernes du Centre-Sud. À ce type correspondent les secteurs dynamiques (biens durables, pétrochimie, chimie, machinerie électrique, acier) fondés sur des procés de production à haute intensité de capital et des forme d'organisation plus modernes (ex., entreprises non familiales, intégrées, diversifiées, etc.).

Ce «déclassement» s'est effectué en deux vagues, dont la première vers la fin des années 50. C'est à Bahia, beaucoup plus que dans l'ensemble du NordEst, que se sont fait sentir les effets caractéristiques de l'expansion industrielle au cours de cette période, à cause de sa proximité du Centre-Sud, de la construction de voies d'accès et de la création du complexe pétrochimique. C'est à Bahia qu'affluaient les investissements du Sud, les entreprises modernes

14. La catégorie propriété familiale est fondée sur le pourcentage d'actions détenu par une famille dans une entreprise. Les entreprises à propriété non familiale sont celles où le capital familial est absent ou ne constitue pas une proportion permettant le contrôle de la firme.

15. La complexité de la technologie est mesurée en déterminant le nombre de techniciens supérieurs et intermédiaires comparativement aux autres entreprises de même taille. Les entreprises se trouvant au dessus de la moyenne relative à chaque catégorie de taille pour chaque catégorie de techniciens ou les deux à la fois ont été classées comme des entreprises à technologie comple$x e$. Celles qui se trouvaient en dessous de la moyenne étaient classées comme entreprises à technologie simple ou non complexe.

16. La taille de l'entreprise était mesurée par le nombre de salariés: Petite $=50-99$ salariés, ordinaire $=100-199$, moyenne $=200-499$, grande $=500-999$, géante $=1000$ salariés et plus .

17. Capital étranger renvoie à toute entreprise dans laquelle on trouve du capital étranger indépendamment du fait qu'il constitue ou non la majorité de la propriété. En fait, seulement $10 \%$ des entreprises étaient sous propriété étrangère.

18. L'origine de classe était mesurée en combinant la profession du père du repondant (mesure objective) avec l'évaluation par le répondant de la situation économique de sa famille au cours de sa jeunesse. Si le père était un grand propriétaire terrien et que la situation économique familiale était prospère, l'individu était classé comme ayant une origine de classe supérieure. Si la situation de la famille était décrite comme pauvre et que le père était un petit commerçant ou un petit fonctionnaire, l'individu était classé comme ayant une origine de classe inférieure. Les autres caractéristiques d'ordre biographique ne sont pas toutes exposées ici. Elles seront examinées dans un essai à venir. 
à forte concentration de capital et les migrants; ainsi, cet État s'intégrait rapidement à l'économie nationale telle que définie par les structures de production et de marché. Il semble que l'ampleur et la rapidité de cette transition expliquent la faiblesse de la bourgeoisie locale (qui n'a pas su s'adapter économiquement à la nouvelle situation, ni manipuler la structure de pouvoir local) comparativement aux industriels d'autres États du Nord-Est.

La seconde vague de développement économique s'est produite vers la fin des années 60 , et résultait, en partie, de l'intégration antérieure de Bahia à l'économie brésilienne dont dépendait, en fin de compte, son redressement économique. En un sens, c'était la continuation de la première phase, si l'on y ajoute le rôle de promoteur joué par le gouvernement dans le système financier, la création de nouvelles banques, la modernisation des opérations dans les banques plus anciennes, ainsi que la création de SUDENE et du programme 34/18. Il ne faut pas sous-estimer l'importance de ces stimulations. Avec l'approbation des agences gouvernementales appropriées, l'expansion d'une entreprise dans le Nord-Est pouvait se faire presque entièrement à partir de prêts, de subventions ou d'autres formes de crédits à même les fonds publics. Parmi les migrants interviewés à Bahia, plus de la moitié (56\%) avaient établi leurs entreprises durant la seconde phase, comparativement au tiers des entreprises autochtones ou étrangères. Les entreprises des migrants se répartissent surtout dans les secteurs les plus modernes exigeant une forte concentration de capitaux, et avec le temps, cela devient de plus en plus vrai. Si bien que certaines observateurs déplorent le développement d'une économie artificielle de moins en moins liée à l'économie traditionnelle en termes $d$ ' «inputs» et d' $d$ outputs $\gg 19$.

La bourgeoisie d'origine étrangère constitue une espèce d'anomalie, car elle correspond aux deux catégories précédentes : 1) la petite industrie familiale et 2) les filiales modernes des transnationales. Les étrangers représentent une part minime de notre échantillon (environ $1 / 5$ ) et comme leur statut est ambigu, nous étudierons surtout les entrepreneurs autochtones de la région ou les migrants. Mais nous analyserons ces trois catégories sous l'angle des caractéristiques de leurs entreprises.

\section{B. OPÉRATIONS DE FINANCEMENT}

Nous avons déjà souligné, certains traits relatifs à l'utilisation importante du crédit bancaire. Les industriels utilisent les banques officielles, telles que la Banque du Brésil et la Banque du Nord-Est, ainsi qu'avec deux ou trois banques privées, notamment celles du Centre-Sud du pays. Nous avons voulu savoir de quelle nature étaient ces transactions, soit l'utilisation des emprunts et les termes clu crédit. Non seulement cela nous éclairera sur la politique de développement de l'État, mais aussi, en ce qui a trait à notre étude, sur les formes de discrimination et de marginalisation. Aussi avons-nous d'abord demandé

19. Goodman a noté le degré remarquablement limité d'interdépendance entre les industries du Nord-Est dans son ouvrage sur le développement dans le Nord-Est, Incentivos a Industrializaçâo e Desenvolvimento do Nordeste, p. 245-250. 
aux industriels qui avaient eu recours au crédit bancaire, de spécifier à quoi ils destinaient ces emprunts de capitaux au cours de l'année précédente. Pour faciliter l'analyse, nous avons ensuite regroupé les réponses en deux catégories selon qu'il s'agissait d'emprunts à court terme ou à long terme.

\section{Emprunts de capitaux à court terme}

À Bahia comme à Pernambuco la majorité des emprunts se faisaient à court terme ${ }^{20}$. Près des trois cinquièmes des industriels $(59 \%)$ avaient emprunté des fonds de roulement et plus du tiers d'entre eux avaient emprunté du capital à court terme. Cela semble indiquer que l'entreprise moyenne dans le Nord-Est a de la difficulté à financer ses dépenses courantes. Parmi les deux plus importants problèmes mentionnés par les industriels concernant leurs entreprises, celui du capital do giro (capital de roulement) revenait souvent. Plusieurs facteurs expliquent ces emprunts fréquents à court terme, notamment la pénurie de capital à long terme ou de capital fixe sur le marché des capitaux. Il semble que les banques, et surtout les banques privées hésitent à consentir des prêts à long terme à de nombreuses industries du Nord-Est. Cela se manifestait à l'égard des secteurs traditionnels, surtout pour les firmes familiales les plus anciennes dont les profits étaient considérablement réduits par un strict contrôle des prix, par une concurrence nationale sévère, par l'inflation et/ou par le pouvoir d'achat réduit de la population. Tous ces facteurs sont particulièrement exacerbés dans le Nord-Est.

Si l'on examine plus attentivement la situation de types particuliers d'industriels, on s'aperçoit que les autochtones qui dirigent, soit des entreprises familiales, soit des entreprises du secteur dynamique sont les plus grands emprunteurs à court terme ${ }^{21}$. On peut en conclure que les premiers, qui constituent le groupe le plus nombreux, sont étouffés graduellement comme l'ensemble de l'économie traditionnelle, et déplacés. La proportion des emprunteurs était de $71 \%$ à Pernambuco comparativement à $85 \%$ à Bahia, ce qui indique les plus grandes difficultés financières de ce groupe, la faiblesse plus grande de ses secteurs et aussi sa plus forte marginalisation ${ }^{22}$. Ainsi que nous l'avons déjà noté, l'économie de Bahia est très imbriquée au marché national (en particulier pétrochimique) alors que Pernambuco, en attirant des investissements dans le secteur des produits de consommation a continué à jouer son rôle «commercial» traditionnel dans la région. Sa bourgeoisie participe donc plus directement au développement de l'économie.

Les autochtones qui dirigent des entreprises dynamiques non-familiales représentent les «déviants» dans notre étude. Plusieurs furent de ces entrepre-

20. Les données tirées d'une étude semblable que j'ai faite à Pernambuco (1970) permettent d'établir certaines comparaisons d'un Etat à l'autre sur les transactions pour le crédit à court et à long terme, mais je n'ai pu obtenir les renseignements sur les taux d'intérêt.

21. D'autres chiffres montrent qu'à Bahia, parmi les autochtones à la tête d'entreprises non familiales dans les secteurs traditionnels, $53 \%$ en sont les fondateurs, $18 \%$ en ont hérité, $12 \%$ sont des propriétaires dirigeants, et $18 \%$ des régisseurs.

22. Goodman et Albuquerque, op. cit, p. 283. Voir également D.E. Goodman, "The Brazilian Economic «Miracle» and Regional Policy, Some Evidence from the Urban Northeast, » Journal of Latin American Studies, 8, 1, mai 1976, p. 2. 
neurs innovateurs décrits par Schumpeter qui, profitant de divers programmes d'industrialisation gouvernementaux (ex. SUDENE, 34/18) quittèrent leurs emplcis dans la fonction publique ou le commerce pour se lancer dans l'industrie. Et leurs nombreux emprunts à court terme reflètent la difficulté qu'ils ont à pénétrer de nouveaux secteurs. Dans ces deux États, les emprunts à court terme dans ces secteurs dynamiques s'accompagnent d'une participation d'intérêts étrangers, ce qui laisse supposer que ces alliances avec le capital étranger sont nécessaires à ces entreprises pour se financer, ou qu'elles sont utilisées par les intérêts étrangers pour pénétrer et profiter du marché financier local.

\section{Emprunts de capitaux à long terme}

Si l'on considère les capitaux empruntés à long terme, on s'aperçoit que comparativement aux migrants et aux étrangers, les autochtones empruntent plus cle capitaux, non seulement à court terme, mais aussi à long terme, du moins en pourcentage. C'est particulièrement le cas à Bahia, ce qui montre que ce groupe est encore plus fragile que son homologue à Pernambuco. Selon d'autres calculs, on voit que les différences les plus accentuées concernent les emprunts de capitaux pour financer de nouvelles installations. Dans ce domaine également, ceux qui font les plus forts emprunts sont les dirigeants des entreprises traditionnelles familiales ou ceux de nouvelles entreprises du secteur dynamique. À Bahia, $86 \%$ des entreprises du premier type et $71 \%$ du second type ont fait des emprunts de capitaux à long terme. À Pernambuco, les chiffres sont respectivement de 61 et de $33 \%$.

Pourquoi ces deux types d'entreprises locales, qui peuvent à peine se financer à court terme augmentent-elles leurs frais en empruntant à long terme? Pour les entreprises familiales, ces gros emprunts de capitaux à long terme peuvent résulter de difficultés temporaires, et de la crainte que leur solvabilité soit menacée par l'émergence de secteurs plus dynamiques. On pourrait, d'une part, interpréter ce phénomène comme une tentative désespérée en vue de surmonter leur insécurité en modernisant rapidement leurs entreprises quel que soit le loyer de l'argent. D'autre part, on pourrait penser que les entreprises familiales traditionnelles ont plus facilement accès au financement à cause de leurs liens avec le système bancaire et qu'elles peuvent obtenir des prêts à longue échéance à des taux d'intérêts raisonnables ${ }^{23}$. Mais tel ne semble pas être le cas. Non seulement leurs liens étaient peu nombreux avec les banques, mais il y avait peu de rapports entre ces liens et les gros emprunts à long terme. Par exemple, à Pernambuco un plus grand pourcentage d'industriels autochtones qu'à Bahia ont indiqué avoir des liens avec les banques ( $55 \%$ comparé à $46 \%$ ) et pourtant la proportion de ceux qui avaient contracté des emprunts de capitaux à long terme était beaucoup plus basse qu'à Bahia (61\% contre $86 \%$ respectivement). En outre, bien que ces deux groupes aient eu des liens surtout avec

23. J'ai estimé qu'il existait des liens entre une entreprise et une banque si l'industriel indiquait a) que lui, ou un membre de la direction de l'entreprise (ou de la société mère), était lié formellement à une banque (comme membre du conseil d'administration par exemple), $b$ ) que son entreprise (ou la société mère) possédait plus que des actions nominales dans une banque, ou c) que la banque participait au conseil d'administration et/ou à la propriété de l'entreprise. 
les banques privées, c'est auprès des banques d'État ${ }^{24}$ que se faisaient surtout les emprunts à long terme à cause de la sécurité plus grande qu'elle offrent. En effet, les banques privées sont prises dans le cercle vicieux des pénuries de capitaux, des taux d'intérêts élevés, de l'inflation et d'un marché capricieux. Elles ne peuvent donc pas faire concurrence aux banques du gouvernement pour ce qui est du volume et des échéances du crédit ${ }^{25}$.

Quels sont alors les rapports entre les types d'entreprises, les banques avec lesquelles les emprunts sont négociés, et les modalités de crédit? Le mode de stratification décrit jusqu'ici ne correspond-il pas finalement à un système d'encouragement/découragement inhérent aux composantes mêmes de la structure financière et orchestré par elle?

\section{MODALITÉS DE CRÉDIT : LE CAS DE BAHIA ${ }^{26}$}

Une des façons de déterminer si un certain groupe d'industriels profite ou non de modalités de crédit favorables pour des catégories particulières d'emprunts, c'est de noter les taux d'intérêt, c'est-à-dire le loyer de l'argent. En se basant sur les chiffres fournis par les industriels, il a été possible de calculer le taux d'intérêt moyen pour chaque catégorie d'emprunt ${ }^{27}$. En général, les variations étaient minimes, et le taux moyen payé par les industriels de Bahia pour les emprunts à long terme était de $17 \%$ par année (ce calcul est basé sur les taux mentionnés par $74 \%$ de ceux qui ont emprunté ce genre de capital). Pour le capital à court terme, le taux moyen était plus élevé, soit $20 \%$ (selon les chiffres fournis par $83 \%$ de ceux qui ont emprunté ce genre de capital). D'autres spécialistes de la politique monétaire brésilienne sont arrivés aux mêmes conclusions. Par exemple, Syvrud a noté que les taux d'intérêt qui s'établissaient entre 10 et $20 \%$ vers la fin des années 60 ont continué à augmenter dans les années 70 pour atteindre des taux annuels qui se situaient entre 21 et $58 \%{ }^{28}$.

Il est intéressant de souligner les raisons de ces fluctuations. Jusqu'en 1964, l'État a permis aux taux d'intérêt d'atteindre le cours du marché, et n'a pas appliqué activement la loi déjà ancienne contre les taux usuraires. Seules, deux banques officielles s'en tenaient au maximum de $12 \%$ permis par la loi, soit la Banque nationale pour le développement économique (BNDE) et la Ban-

24. Même à Bahia, les liens avec les banques ne semblent pas expliquer la facilité d'accès au crédit à long terme. Les nouveaux arrivants, même liés à des banques privées, ont plutôt tendance à demander des prêts aux banques du gouvernement.

25. Pour obtenir des renseignements détaillés sur l'intervention de l'État dans le système bancaire et financier, voir Wilson Suzigan et al., Financiamento do Projectos Industriais no Brazil, p. $41-80$ et $92-101$.

26. Comme l'étude sur Pernambuco ne comportait pas de données sur les taux d'intérêt sur les emprunts à court et à long terme, l'analyse suivante portera uniquement sur Bahia.

27. Selon un spécialiste du système monétaire brésilien: «Aucun banquier n'admettrait jamais qu'il prête à des taux supérieurs à ceux qui sont fixés par le Conseil monétaire national (du Brésil). Voir Donald E. Syvrud, «Estrutura e Politica de Juros no Brazil - 1960/1970», Revista Brasileira da Economia, 26, 1, janvier-mars, 1972, p. 121.

28. D'après Syvrud, dont les chiffres étaient basés sur une étude résultant d'une enquête de l'Association commerciale de Rio de Janeiro auprès d'une cinquantaine d'industriels. Donald Syvrud, ibid. p. 120. 
que du Brésil. Toutefois, comme les taux d'intérêt subissaient une augmentation parallèle à celle des prix, l'État s'est inquiété de cette poussée inflationniste et de la tendance des institutions financières privées à octroyer du crédit en fonction «...des relations familiales des clients plutôt que de leur solvabilité " ou de la valeur des projets. Comme cela créait de graves déséquilibres dans l'économie et particulièrement dans les régions en voie de développement (ex., le Nord-Est), le gouvernement a décidé de jouer un rôle plus actif sur le marché financier ${ }^{29}$. Les secteurs et les projets prioritaires ont bénéficié de taux d'intérêt préférentiels et de crédit sous forme de subventions, grâce à la Banque du Brésil et à la BNDE.

Malgré le laxisme de l'État dans l'application de sa loi contre l'usure, les taux d'intérêt excédaient rarement le taux de l'inflation, ce qui signifie que la plupart des prêts se faisaient à un taux réel négatif. $\grave{A}$ cause d'une inflation galopante, en 1964, les prêts des banques commerciales atteignaient un taux négatif cle $30,1 \%$ et ceux de la Banque du Brésil, un taux négatif de $37,5 \%$. Cette politique (ou ce manque de politique) entraîna les conséquences suivantes: a) la croissance d'un système financier intermédiaire très coûteux et la prolifération d'institutions financières, d'agences bancaires et de compagnies de prêts $; b$ ) une concurrence féroce entre les banques pour attirer l'épargne du public, ce qui entraîna des coûts de fonctionnement et d'administration exorbitants, et $c$ ) la bousculade des industriels pour obtenir des prêts subventionnés (crédit à bon marché) plutôt que d'utiliser leurs propres ressources (ou surplus de capitaux) qu'ils investissaient dans l'immobilier, dans d'autres valeurs ou dans des devises étrangères. Les industriels empruntaient des fonds pour remplacer leur capital de roulement qui se dévaluait sous l'effet de l'inflation. Notre enquête révèle qu'ils passaient une bonne partie de leur temps à la praça (Bourse commerciale/financière), allant d'une banque à l'autre, pour tenter d'emprunter des capitaux au taux le plus bas possible. Si l'on tient compte de la centralisation de la direction des entreprises dans le Nord-Est, il n'est pas douteux que l'absence du patron devait porter préjudice à ses affaires.

Entre 1964 et 1969, l'État a tenté de corriger la distorsion des taux d'intérêt en utilisant des mesures monétaires et des subventions, mais sans grand succès. Malgré les subventions aux banques commerciales - que la plupart acceptèrent - elles continuèrent à bénéficier d'un intérêt moyen de $30 \%$ sur leurs prêts. Cette mesure s'avéra aussi inefficace que la loi contre l'usure pour réduire les taux d'intérêt. En 1970, l'État adopta une nouvelle politique. Il supprima les plafonds sur les taux d'intérêt imposés aux banques commerciales en espérant que la concurrence contribuerait à réduire les taux d'intérêt, et que la structure financière serait rationalisée ${ }^{30}$. Il semblait que l'État abandonnait son rôle d'intermédiation, alors qu'en réalité il prenait une nouvelle orientation. Il établissait graduellement son hégémonie sur toute la structure financière du pays, et ce faisant excluait certaines classes de certaines formes de crédit.

29. Donald E. Syvrud, Foundations of Brazilian Economic Growth, Stanford, California, Hoover Institution Press, 1974, p. 96 et 104. L'analyse qui suit est basée, en grande partie, sur le chapitre intitulé : «The Impact of Interest Rate Policy ", surtout, p. 100-117.

30. Ibid. p. 111-117. 
Si l'on différencie les industriels on constate que les autochtones payent des taux d'intérêt plus élevés pour les emprunts à long terme, et plus bas pour les emprunts à court terme. Pour les migrants et pour les étrangers, c'est l'inverse. Précisons que le taux d'intérêt moyen payé par les autochtones pour les emprunts à long terme était de $16,6 \%$ par année, comparativement à $15,9 \%$ payé par les migrants. Pour les emprunts à court terme, les autochtones payaient $19,3 \%$, les migrants $20 \%$ et les étrangers $21 \%$ par année. Ces chiffres indiquent que pour l'emprunt de capitaux à long terme - très importants pour l'expansion industrielle - les autochtones ne bénéficiaient pas de conditions aussi favorables que les autres industriels. Pour savoir si cela est particulier à l'entreprise locale ou à un secteur industriel donné, à une forme de propriété, à la taille de l'entreprise ou à sa complexité technologique, il vaut mieux considérer la proportion de ceux qui payaient des taux d'intérêt plus ou moins élevés que la moyenne à court terme ou à long terme. Bien que nos conclusions soient approximatives à cause du petit nombre d'industriels qui ont révélé leurs taux d'intérêt, un certain schéma se dégage néanmoins de nos données.

Si nous étudions la bourgeoisie industrielle selon la forme de propriété, nous notons que les entreprises familiales payaient des taux d'intérêt plus élevés que la moyenne pour les emprunts à long terme que la plupart des entreprises non familiales. Le même schéma vaut pour les petites entreprises comparées aux grandes, pour les entreprises indépendantes comparées aux filiales et en termes de classes sociales, quand des industriels originaires de la grande bourgeoisie sont comparés à ceux de la classe moyenne. Mais c'est en tenant compte des diverses caractéristiques d'une entreprise que l'on comprend mieux le processus de marginalisation qui s'est produit à Bahia.

Voyons d'abord l'entreprise familiale et le secteur industriel. Comme on l'a déjà vu, une plus grande proportion d'entreprises familiales payaient des taux d'intérêt plus élevés que les entreprises non familiales. Mais c'est particulièrement le cas des entreprises familiales dans les secteurs dynamiques où presque tous les industriels payaient des taux supérieurs à la moyenne. Et cette différence se manifeste particulièrement parmi les autochtones. Alors que $58 \%$ des entreprises familiales autochtones du secteur traditionnel payent des taux plus élevés, la proportion des entreprises familiales du secteur dynamique atteint dans ce cas $86 \%$. Sur la base de nos données, on voit que l'augmentation n'est pas aussi marquée en ce qui concerne les entreprises des migrants. En fait, d'autres calculs indiquent que si l'on tient compte seulement des taux d'intérêt pour le capital d'équipement - l'élément principal des emprunts à long terme - alors les migrants dans les secteurs dynamiques ont payé des taux inférieurs à la moyenne, alors que pour les autochtones, la tendance demeure inchangée. Ce qui semble s'être produit à Bahia (ou ce qui s'est produit) c'est que les entreprises familiales qui se sont aventurées dans les secteurs dynamiques ont dû payer des taux d'intérêt plus élevés, ce qui les a pénalisées, et les a empêchées de proliférer dans ces secteurs. Cependant, c'est le contraire qui s'est produit pour les dirigeants autochtones d'entreprises non familiales, particulièrement dans les secteurs dynamiques. En fait, pour ce groupe (autochtone, dynamique, non familial) les taux d'intérêt sont plus bas que pour tous les autres groupes, ce qui laisse supposer qu'on encourage ce groupe à étendre ses activités. 
Cependant, même si l'on a empêché certains industriels autochtones de moderniser et d'agrandir leurs usines, ils ne sont pas complètement marginalisés. Les banques semblent leur permettre de continuer clopin-clopant en leur accordant plus de prêts à court terme et à des taux d'intérêt favorables. C'est particulièrement le cas pour les autochtones qui, dans les entreprises familiales, ont bénéficié de prêts à court terme dans une plus grande proportion que les autres ( $78 \%$ ), mais qui, en moindre proportion (36\%), ont payé des taux d'intérêt plus élevés que la moyenne. Ceci est particulièrement apparent dans les secteurs traditionnels et dans le cas des autochtones de la grande bourgeoisie dans ces secteurs. Le propriétaire d'une entreprise de corroyage se plaignait de l'attitude de la banque d'État pour le développement régional, la Banque du Nord-Est, «qui nous traite, disait-il, de façon très cavalière, qui n'a jamais délégué quelqu'un pour visiter nos installations, ni analysé sérieusement notre secteur. Mais je ne peux pas me plaindre de la Banque du Brésil qui a toujours répondu par un « oui » ou par un «non» à nos demandes d'emprunts de capital de roulement.»

Qu'en est-il des deux autres groupes d'industriels, les migrants et les étrangers? Comment leur situation se compare-t-elle à celle des autochtones?

D'après notre enquête, les nouveaux arrivants et les étrangers payent des taux d'intérêt plus élevés que la moyenne sur les prêts à court terme. Ces prêts étaient consentis en majorité par la Banque du Brésil dont le rôle officiel pouvait être de limiter l'accès des entreprises du secteur dynamique au crédit à bon marché, ce qui leur permettait de transformer en actif liquide leur surplus de capital ou leurs investissements ${ }^{31}$. Mais il est plus vraisemblable que la politique de la Banque du Brésil ait été de réserver au secteur traditionnel les prêts à coult terme. Ainsi, en 1970 la plus grande partie de ses prêts (39\%) est allée à l'alimentation, aux textiles et au vêtement pour aider ces producteurs à payer leurs frais de roulement ${ }^{32}$.

Cependant, pour les emprunts à long terme, la tendance était inverse. La banque semblait favoriser certains industriels ${ }^{33}$, notamment les migrants dans le secteur dynamique, qui dirigeaient de grandes entreprises non familiales d'une technologie avancée. La tendance était de leur consentir des prêts à long terme, à des taux inférieurs au taux d'intérêt moyen. De plus, si l'on ne tient comple que des emprunts pour de nouvelles installations, alors les migrants de cette catégorie sont les industriels «les plus favorisés» en ce qui concerne le coût des emprunts à long terme. En outre, beaucoup de ces entreprises pou-

31. La politique des taux d'intérêt d'avant 1964 a eu pour effet, de déformer la structure financière des sociétés brésiliennes. Le coût du capital emprunté était moins élevé que celui du capital ordinaire (equity capital) ce qui permettait l'expansion des firmes ayant accès à ce crédit à bon marché. Le surplus de capital était investi dans l'immobilier et dans d'autres valeurs qui ne se dépréciaient pas avec l'inflation. Après 1966, les taux d'intérêt qui étaient négatifs sont devenus hautement positifs, ce qui entraîna beaucoup de faillites et entrava les efforts de stabilisation. Voir Donald Syvrud, Foundations of Brazilian Economic Growth, p. 103-104.

32. Wilson Suzigan et al., Financiamento do Projectos Industriais no Brasil, p. 146-150.

33. L'analyse ne portera pas sur les industriels étrangers, attendu que seulement cinq d'entre eux ont fait état des taux d'intérêt sur les prêts à long terme. Cela s'explique par la concentration des opérations financières de leurs sociétés dans le Centre-Sud, ou au siège social de la compagnie mère. 
vaient être liées aux entreprises étrangères du Centre-Sud dont la réputation financière est plus solide. Il ne faut pas oublier cependant que la majorité de ces prêts ont été consentis par la Banque nationale de développement (BNDE). Apparemment, le gouvernement encourageait l'établissement de ce type d'entreprises dans le Nord-Est.

Par contre, les taux d'intérêt étaient plus élevés pour les migrants œuvrant dans les secteurs traditionnels, et liés à des entreprises familiales de petite taille, d'une technologie simple et ne bénéficiant d'aucune affiliation, quelle que soit l'origine de classe de ces industriels. Le fait de limiter notre analyse aux emprunts pour de nouvelles installations ne change pas grand chose à nos conclusions. Les deux tiers des migrants qui dirigeaient des entreprises familiales traditionnelles ont payé plus que le taux moyen pour les emprunts à long terme, et dans les secteurs dynamiques la proportion est encore plus élevée, comme elle l'est pour les autochtones. Toutefois, ces migrants ne bénéficient pas, comme les autochtones, d'une compensation touchant leurs emprunts à court terme. D'autres calculs indiquent que les plus défavorisés pour ce qui est des emprunts à court terme, ce sont les migrants de la bourgeoisie cuvrant dans de grandes entreprises familiales liées à des compagnies du Centre-Sud. En fait, les entreprises de ce type ont été l'objet d'une double discrimination pour les emprunts à court et à long terme.

Alors qu' on «envoyait paître» les industriels autochtones des entreprises familiales, on décourageait complètement, sur le plan financier, le migrant propriétaire d'une entreprise familiale. Ce dernier groupe rappelle les «carpetbaggers» du Nord qui s'infiltraient dans le Sud des États-Unis après la guerre de Sécession, pour s'emparer des biens immobiliers, des marchés et d'une économie ruinée.

Jusqu'ici, nous avons négligé le rôle du capital étranger en étudiant les modalités de crédit ${ }^{34}$. Maintenant, posons la question suivante : Est-ce que la participation du capital étranger dans les entreprises industrielles du Nord-Est a un effet favorable ou défavorable sur les taux d'intérêt?

Comme dans l'analyse des autres caractéristiques d'entreprise, la réponse à cette question implique une analyse des types industriels en liaison avec le secteur industriel et la propriété familiale.

Les chiffres concernant les emprunts à long terme des autochtones indiquent que les banques semblent défavoriser les entrepreneurs locaux qui s'associent au capital étranger ${ }^{35}$. Ceci est tout à fait apparent dans les secteurs dynamiques et la discrimination s'étend aux emprunts à court terme. Par ailleurs, les entreprises autochtones traditionnelles qui fonctionnent sans participation du capital étranger obtiennent des taux d'intérêt favorables pour le long

\footnotetext{
34. Ici, nous ne nous intéressons qu'à la participation du capital étranger. La propriété étrangère de l'entreprise fera l'objet d'une autre étude.

35. Il est possible que des entreprises autochtones s'associent à des intérêts étrangers simplement pour obtenir le capital dont elles ont grandement besoin. En ce cas, la discrimination gouvernementale serait motivée par l'insolvabilité de l'entreprise. Soulignons cependant que la participation du capital étranger ne semble pas régler le problème du capital à court terme nécessaire à l'entreprise.
} 
terme et le court terme. Par exemple, seulement $28 \%$ de ces derniers ont payé des taux d'intérêt plus élevés que la moyenne sur les prêts à court terme, comparativement à $75 \%$ des autochtones des secteurs dynamiques dirigeant des entreprises à participation de capital étranger. Selon nos données, les banques semblent avoir exercé une discrimination surtout vis-à-vis des entreprises familiales autochtones associées au capital étranger. La plupart ont dû payer des taux d'intérêt plus élevés que la moyenne pour leurs emprunts à long terme et à court terme. Les institutions financières de Bahia et Pernambuco ne semblent pas favorables à l'alliance du capital étranger et du capital familial. L'alliance entre le capital familial autochtone dans les secteurs traditionnels et les sociétés financières brésiliennes du Centre-Sud semble plus acceptable. Mais les plus favorisées sont les entreprises non familiales qui ont des liens de parenté étrangers, quelle que soit l'origine géographique des industriels.

\section{LES AGENTS DE MARGINALISATION : LES BANQUES}

Nous avons identifié, parmi la bourgeoisie de Bahia, les industriels qui ont été marginalisés et ceux qui ont été favorisés, mais nous avons à déterminer quels furent les principaux agents de cette marginalisation. On peut à partir de nos données identifier la proportion des divers bénéficiaires de prêts à long et à court terme, mais aussi les principaux prêteurs de capitaux. Ainsi, par exemple, la grande banque nationale, la Banque du Brésil, a été la principale source de capital argent pour l'industrie «traditionnelle», l' «entrepreneurship» autochtone, le capitalisme familial et la classe supérieure; par ailleurs, les banques de développement, du Sud ou de la région ont surtout financé l'industrie «moderne», les migrants, la classe moyenne et l'entreprise non familiale. Mais au-delà de ces rapports privilégiés entre certaines banques et certains industriels, il nous reste à découvrir si l'apparente division du travail entre les différents types de capitaux répartis entre divers groupes correspond à une différenciation des taux d'intérêt.

Le rôle marginalisateur des banques se manifeste particulièrement dans le cas des autochtones issus de la classe supérieure dirigeant des entreprises familiales dans les secteurs dynamiques et les migrants propriétaires d'entreprises familiales dans les secteurs traditionnels (les «carpetbaggers»). Nos données montrent que les principaux bailleurs de fonds pour ces groupes ont été la Banque clu Brésil et celle du Nord-Est, et dans le cas des migrants, des banques privées du Sud également. Ces banques imposaient des taux d'intérêt plus élevés aux autochtones de la classe supérieure sur les prêts à long terme, surtout s'il s'agissait d'entreprises familiales dans des secteurs dynamiques. Notons, à ce sujet, que les quelques autochtones issus de la classe supérieure qui ont pu obtenir des prêts de banques d'État ou de développement national, ont dû payer des taux d'intérêt plus élevés que la moyenne. Cependant si l'on examine les prêts à court terme on s'aperçoit que la classe supérieure est traitée de façon plus favorable par le gouvernement. Nombre d'entre eux ont non seulement obtenu des prêts de ce type, mais les taux d'intérêts étaient plus bas que la moyenne. La bourgeoisie non locale (migrants et étrangers) étant très limitée, on ne peut que faire une hypothèse à son sujet, mais j'estime que la propriété 
familiale et le secteur industriel sont deux facteurs plus importants dans la distribution du crédit que la classe sociale d'origine. Évidemment, le secteur traditionnel, la propriété familiale et la classe supérieure sont des éléments intimement liés.

La situation des migrants qui dirigent des entreprises familiales traditionnelles est comparable, du moins en ce qui concerne les emprunts à long terme. En outre, comme nous l'avons vu, sur les emprunts à court terme, un taux d'intérêt plus élevé leur était imposé, non seulement par les banques d'État, mais aussi par les banques privées locales et par celles du Sud. Il semble donc que ce groupe soit le plus défavorisé dans le Nord-Est, peut-être parce qu'il menace la position des entrepreneurs locaux déjà marginalisés par des nouveaux arrivants dynamiques.

Il y a les industriels marginalisés, mais il y a aussi ceux qui ont bénéficié de taux d'intérêt favorables de la part des banques; par exemple, les autochtones à la tête de grandes entreprises ou les autochtones dans les entreprises non familiales ceuvrant dans des secteurs traditionnels, ou les migrants et les directeurs étrangers d'entreprises non familiales dynamiques. Quelles sont alors les banques qui prêtaient à des taux favorables?

Ce sont les banques d'État qui ont joué ici le principal rôle, et particulièrement la banque de développement régional, la Banque du Nord-Est, qui a octroyé la plupart des prêts à long terme et à taux d'intérêt inférieurs à la moyenne aux autochtones dans les grandes entreprises, particulièrement dans les secteurs traditionnels. En fait, c'est cette banque qui leur a consenti plus de $60 \%$ de leurs emrpunts à long terme. Et c'est la Banque du Brésil qui a octroyé à ce même groupe la plus grande proportion des prêts à court terme et à des taux favorables.

C'est la BNDE, la Banque nationale de développement du Brésil, qui a fourni du crédit aux migrants, du moins à ceux qui œuvraient dans les secteurs dynamiques, dans les grandes entreprises et dans les compagnies non familiales. Pourtant, cette banque est rarement mentionnée comme source de financement par les autochtones, et jamais par les étrangers. Pour leur capital de roulement, les industriels de ce dernier groupe obtenaient des prêts des banques commerciales du Sud à des taux d'intérêt favorables.

Il est donc évident que les banques d'État jouent un rôle extrêmement important, plus important même que les banques commerciales, non seulement dans le financement, mais aussi dans la stratification des entreprises industrielles de Bahia. Le pouvoir de sanctionner que possède la Banque du Brésil se révèle très important. Il a servi à marginaliser complètement les migrants dans les secteurs traditionnels; partiellement les autochtones à la tête d'entreprises familiales. Par contre, il a servi à endosser les grandes entreprises autochtones non familiales.

La Banque du Nord-Est joue également un rôle dans la stratification industrielle de la région en encourageant les entrepreneurs locaux, grâce à des prêts à long terme et à taux d'intérêt favorables. Elle finance notamment les grandes entreprises traditionnelles non-familiales dirigées par des autochtones. 
Dans une moindre mesure, cette banque fait aussi des prêts à court terme, mais comme ses taux sont en général supérieurs à la moyenne, elle n'endosse pas un groupe particulier d'une façon aussi décisive que la Banque du Brésil. Ceci est probablement dû à la récente expansion de ses activités dans le secteur commercial, au lieu de s'en tenir à son rôle de banque d'investissement ou de développement.

Les autres banques du gouvernement jouent un rôle secondaire, mais important, néanmoins, pour certains groupes. La banque de développement de l'État de Bahia, la Desenbanco a apporté son aide aux petits industriels autochtones en leur donnant accès au crédit à long terme à des taux favorables pour leurs entreprises non familiales à haute technologie. À ce sujet, je me rappelle que le président de la banque, en m'exposant sa politique de prêt a mentionné les critères suivants : le secteur industriel, la «solidité du groupe» (son bilan, ses investissements), les références bancaires, le «savoir-faire», la structure administrative et les chances de succès. Et il a continué en disant :

Desenbanco n'endosse ni les monopoles, ni un émiettement de la production afin de ne pas créer de potentiel non utilisé (capacidade ociosa). Je dois ajouter que ceci est également la politique de SUDENE. Nous accordons la priorité aux entreprises chimiques, pétrochimiques, métallurgiques, minières, agro-industrielles et au tourisme. Il nous est plus difficile d'accorder des crédits à des secteurs tels que l'industrie forestière, les breuvages, les huiles végétales et animales et le meuble. Nous délaissons les secteurs de l'économie familiale et traditionnelle, pour favoriser une économie d'entreprises modernes et capitaliste. Ici, au Brésil, dans l'industrie, le propriétaire (dono) vit avec faste et les travailleurs misérablement. À cause de cela, le gouvernement brésilien les force à participer aux obligations sociales. Cette participation diminuera s'ils changent de mentalité. C'est une mesure disciplinaire (pour l'industrie). Toutes les entreprises industrielles doivent rapporter à l'industrie et fournir des bénéfices sociaux.

Si une entreprise est en difficulté, nous essayons de l'aider. C'est notre politique, et nous encourageons l'entrepreneur à s'associer ou à céder ses actions à une autre entreprise. Ainsi, nous récupérons les dettes, et nous pouvons prêter au nouveau groupe, de préférence après le transfert du contrôle.

Si l'entreprise n'accepte pas de faire cela, évidemment, nous opérons une saisie.» (Interview avec Arthur Ferreira, président de Desenbanco, 22 mai, 1974.)

La Banque nationale de développement (BNDE), ainsi que nous l'avons déjà noté, favorisait d'abord les migrants en offrant du crédit à long terme et à intérêt réduit à ceux qui œuvraient dans de grandes entreprises à technologie avancée, dans les secteurs dynamiques. Comme cette banque ne fait pas de prêts à court terme, son appui financier n'est pas aussi complet que celui de la Banque du Brésil. Plusieurs observateurs estimént, néanmoins, qu'elle est, potentiellement, le moyen le plus puissant pour attirer les investissements du CentreSud vers le Nord-Est, mais il ne s'agirait pas d'investissements étrangers, du moins pas directement. D'ailleurs, nos calculs révèlent qu'après le capital familial, c'est le capital étranger que les banques officielles favorisent le moins. Quand il y a participation de capital étranger dans les entreprises autochtones, 
elles paient des taux d'intérêt plus élevés que la moyenne sur les prêts à court et à long terme. Il faut noter, toutefois, que des banques privées locales, comme la Banco Economico, ont endossé l'alliance du capital autochtone et étranger, sous forme de crédit à court terme à des taux d'intérêt favorables. La BNDE et des banques privées du Sud encouragent également les rapports entre le capital étranger et celui des migrants. De même, à partir de cas peu nombreux, on peut conclure que Desenbanco, selon la politique exprimée par son président, favorise certaines alliances entre le capital étranger et celui des nouveaux arrivants dans des filiales de compagnies du Centre-Sud dirigées par des étrangers.

Pour résumer, notre étude démontre à l'évidence que les banques d'État, en utilisant des taux d'intérêt différentiels, encouragent certains groupes, en découragent d'autres, en laissent subsister certains, et amalgament lentement différents intérêts et différentes classes.

\section{RÉSUMÉ ET CONCLUSIONS}

Il est évident que les institutions financières, au Brésil, en manipulant le crédit transforment la structure et la stratification industrielles à Bahia, Pernambuco et ailleurs dans le Nord-Est. J'ai montré qu'on encourage fortement certains industriels qui, par le fait même, prospèrent : les gestionnaires migrants ou étrangers œuvrant surtout dans de grandes entreprises non familiales, à haute technologie et dans des secteurs dynamiques. D'autres sont marginalisés, ou « on les envoie paitre»: les autochtones ou les industriels locaux liés aux entreprises familiales traditionnelles. Enfin, d'autres sont complètement défavorisés et exclus du marché : les autochtones dont les entreprises familiales se son aventurées dans les secteurs dynamiques, ou les migrants et les étrangers qui ont établi des entreprises dans le secteur traditionnel. Certes il y a des anomalies, des cas particuliers. On pourra leur faire payer des taux d'intérêt élevés, malgré l'aval de SUDENE. Cette stratification sociale entraîne l'émergence d'une structure de classe industrielle claire et rigide, même en cette période mouvante, à Bahia. Et à Pernambuco, dont je n'ai pas beaucoup parlé, la même stratification s'est produite.

Les agents de ces changements, en même temps que les principaux facteurs de ces divisions, sont les banques gouvernementales, particulièrement la Banque du Brésil (nationale), et la Banque du Nord-Est (banque régionale de développement). À cet égard, j'ai noté que la Banque du Brésil joue trois rôles. Avec le concours de la Banque du Nord-Est, elle soutient ou marginalise partiellement : c'est le cas des autochtones dans les entreprises familiales traditionnelles; elle décourage ou marginalise complètement, comme dans le cas des migrants dans les entreprises familiales traditionnelles, ou elle donne son appui, comme dans le cas des industriels autochtones affiliés à des entreprises nonfamiliales. Aucune autre banque d'État ne joue des rôles aussi variés dans le secteur financier. Les autres banques, qu'elles soient gouvernementales ou privées, servent des clientèles spécifiques, et se spécialisent dans le crédit à long ou à court terme. Ceci est un autre signe de la division du travail et de la stratification qui caractérisent Bahia. À ce sujet, on a noté que la Banque du 
Brésil se consacre surtout au financement à court terme de l'entrepreneurship local, du capitalisme familial et des secteurs traditionnels, alors que la Banque du Nord-Est fait surtout des prêts à long terme et à des taux favorables aux entrepreneurs autochtones, à condition qu'ils soient dans des entreprises non familiales. Quant à la banque nationale de développement (BNDE), elle endosse les migrants dynamiques en leur fournissant du crédit à long terme à des taux intéressants, surtout, semble-t-il, s'il y a participation du capital étranger. La division du travail se prolonge donc au niveau de l'État. Nous avons vu que cela se vérifie pour ce qui est de l'élite étrangère. Alors que la Banque de développement de l'État de Bahia (Desenbanco) apparaît comme la première source de crédit à long terme pour les grandes filiales de compagnies étrangères, la banque d'État (BANEB) fournit du capital à long terme aux plus petites entreprises indépendantes. Et l'on voit que les banques privées locales fournissent du capital à court terme aux étrangers dans les secteurs traditionnels et aux autochtones qui ont fait alliance avec le capital étranger.

Sous un autre angle, on voit apparaître un réalignement des fractions de classe. Au niveau de l'État, se crée une alliance directe avec le capital étranger, et il semble que la bourgeoisie financière locale - dont certains représentants sont les héritiers de l'oligarchie agro-commerciale - tente d'incorporer les étrangers. Cette nouvelle politique de participation «a pour objectif avoué d'établir un rapport de forces «plus équitable» entre les intérêts locaux et le capital étranger, sans recourir aux mesures classiques de l'expropriation, ou à la délirnitation de secteurs de production réservés aux nationaux ${ }^{36}$. Aux niveaux national et régional, la situation n'est pas claire. La banque nationale de développement endosse la participation du capital étranger, mais d'une façon indirecte (par l'intermédiaire des migrants) et restreinte (aux entreprises non familiales dans les secteurs dynamiques). La Banque du Brésil semble défavoriser l'alliance du capital étranger avec la classe traditionnelle locale par le truchement de ses taux de crédit élevés. En réfléchissant à cette configuration, on se demande si cette division du travail ou l'étroite corrélation entre certaines institutions financières et certains industriels est le résultat d'une stratégie consciente de la part du régime pour maintenir une structure de classe «Équilibrée » et rigide à une époque caractérisée par la fluidité et le dynamisme économique. On peut se demander pourquoi le régime, au moyen de ses banques, ne coupe tout simplement pas les vivres aux entreprises familiales traditionnelles qui survivent à peine grâce à des injections périodiques de capital à court terme à des taux favorables. La réponse à ces questions se trouve en partie dans la «philosophie» du régime telle qu'exprimée par ses porte-parole (par ex. le président de Desenbanco) quand ils discutent de l'intérêt de «discipliner» les récalcitrants et de récupérer les faillites. D'une part, les entreprises familiales semblent odieuses au régime, parce qu'elles symbolisent l'archaïsme, une influence et un pouvoir embarrassants, la désorganisation et un capitalisme

36. Luciano Martins, «La joint-venture Etat - Firme transnationale - Entrepreneurs locaux au Brésil» dans ce même numéro, p. 170. Voir également Peter Evans, "Multinationals, State-Owned Corporations, and the Transformation of Imperialism: A Brazilian Case Study », Economic Development and Cultural Change, 26, 1, octobre, 1977; et son dernier livre, Dependent Development of the Alliance of Multinational State and Local Capital in Brazil, Princeton, N.J., Princeton University Press, 1979. 
sans entrave; d'autre part, on sent que le régime désire les intégrer dès qu'elles participent au «miracle économique» et qu'elles endossent la «révolution nationale ». Ainsi, il est curieux de constater que le coronelismo, qui fut à une époque le premier mécanisme de contrôle social de l'oligarchie agro-commerciale est maintenant entre les mains de la classe moyenne des technocrates brésiliens, et est utilisé contre ses créateurs pour leur imposer les normes d'un capitalisme « rationalisé ».

D'autres faits laissent croire à une politique délibérée de la part du régime. Une analyse du crédit à court terme et à long terme, et des modalités de prêts, ainsi que de la division du travail entre les banques nous montre que chacun reçoit quelque chose et que personne n'est exclu. Mentionnons, à titre d'exemples, la récente prolifération des banques officielles de développement (particulièrement au niveau régional), les changements survenus à SUDENE pour apaiser les revendications des économies traditionnelles, la récente percée des banques commerciales locales dans le champ des investissements et la politique relativement tolérante (permissive) du régime envers le capital étranger. L'analyse de cette configuration financière à Bahia semble montrer que la volonté du régime est de contribuer à l'émergence d'une nouvelle classe industrielle et d'affaiblir l'ancienne. C'est comme s'il tentait d'équilibrer la structure politique, tout en revitalisant l'économie, en imposant l'Ordem aux groupes traditionnels, et en offrant aux modernes le Progresso.

\section{RÉSUMÉ}

Cet article constitue une étude empirique des pratiques discriminatoires imposées par les banques à la bourgeoisie industrielle et à leurs entreprises dans l'État du Nord-Est du Brésil qui se développe aujourd'hui le plus vite. Fondé sur une enquête par interviews auprès d'un échantillon aléatoire et systématique des dirigeants des plus grandes entreprises, ainsi que sur des données statistiques provenant des archives gouvernementales et bancaires cet essai établit l'histoire économique et politique de cet État et les problèmes qui ont entravé son émergence. Une analyse des opérations de crédit, des emprunts à court et à long terme, et de taux d'intérêts montre que les banques d'État, en particulier la Banque du Brésil, marginalisent la bourgeoisie industrielle associée aux secteurs économiques traditionnels et aux entreprises familiales. Elles appuient, par contre, les dirigeants des firmes nouvelles, à haute intensité de capital qui sont souvent des filiales de grands conglomérats nationaux ou internationaux. L'une des conséquences inattendues de l'intervention de l'État et de l'hégémonie du capital financier est la pénétration du capital étranger dans les secteurs à haute intensité de capital. Ceci peut éroder le soutien et le sacrifice populaires sur lesquels le régime compte pour poursuivre son miracle économique. Cet essai montre ainsi comment les institutions clés sont utilisées par l'État pour stratifier et contrôler les classes sociales dans une des zones au développement le plus rapide en Amérique latine et peut être dans le Tiers Monde.

\section{SUMMARY}

This is an empirical study of discriminatory practices imposed by banks on the industrial bourgoisie and their enterprises in Bahia, the most rapidly developing state in the Northeast of Brazil. Based on a systematic, random (interview) survey of the largest manufacturers and statistical data from banking and governmental archives, the essay establishes the political and economic history of the state and the problems which hindered its emergence. An analysis of credit transactions, short and 
long term borrowing, and interest rates shows that state banks, particularly the Bank of Brazil, marginalized the industrial bourgoisie associated with traditional economies and family-owned enterprises but endorsed those heading the newer, capital-intensive, often subsidiary of a larger national or international conglomerate. One unintended consequence of state intervention and hegemonization of finance capital is the penetration of foreign capital into labor intensive sectors. This could erode the popular support and sacrifice on which the regime relies to continue its economic miracle. Here is an essay which illustrates how key institutions are used by the State to stratify and control social classes in the most rapidly developing areas in Latin America, if not the Third World.

\section{RESUMEN}

Este articulo constituye un estudio empirico de las practicas discriminatorias impuestas por las bancas a la burguesía industrial y a sus empresas en el Estado del Nord Este del Brasil que hoy día se desarrolla rapidamente. Fundado sobre una encuesta por entrevistas segun una muestra aleatoria y sistemática de dirigentes de las empresas más grandes, asi que sobre datos estadísticos que vienen de los archivos guvernamentales y bancarios, este ensayo establece la historia económica y politica de este Estado y los problemas que han estorbado su emergencia. Un análisis de las operaciones de crédito, de prestamos a corto y largo plazo, y las tasas de intéres, muestra que las bancos del Estado, en particular El Banco del Brasil, marginalisan la burguesía industrial asociada a los sectores economicos tradicionales y a las empresas familiares. Ellas apoyan en cambio los dirigentes de nuevas firmas, a alta intensidad de capital que son a menudo filiales de los más grandes conglomerados nationales o internacionales. Una de las consecuencias inesperadas de la intervención del Estado y de la hegémonia del capital financiero es la penetración del capital estranjero en los sectores a alta intensidad de capital. Esto puede corroer el apoyo y el sacrificio popular sobre los cuales el régimen cuenta para continuar su milagro económico Este ensayo muestra de esta rnanera como las instituciones claves son utilisadas por el Estado para estratificar y controlar las clases sociales en las zonas de desarrollo más intensas en América-Latina y a lo mejor del Tercer Mundo. 